ИЗВЕСТИЯ АҚАДЕМИИ НАУК ЭСТОНСКОИ ССР. ФИЗИКА * МАТЕМАТИКА PROCEEDINGS OF THE ACADEMY OF SCIENCES OF THE ESTONIAN SSR. PHYSICS * MATHEMATICS

$1989, \quad 38,2$

\title{
КОЛЕБАНИЕ СВЕРХСТРУКТУР С УПОРЯДОЧЕННЫМИ КИСЛОРОДНЫМИ ВАКАНСИЯМИ В РЕШЕТКЕ $\mathrm{YBa}_{2} \mathrm{Cu}_{3} \mathrm{O}_{7-x}$
}

\author{
(Представил К. К. Ребане)
}

Для сверхпроводящих кристаллов со стехиометрией $\mathrm{Y}_{2} \mathrm{Ba}_{4} \mathrm{Cu}_{6} \mathrm{O}_{13}$ (орто-II-фаза) и $\mathrm{Y}_{8} \mathrm{Ba}_{16} \mathrm{Cu}_{24} \mathrm{O}_{55}$ вычислены симметризованные колебания в Г-точке зоны Бриллюэна. Обсуждаются изменения в спектрах КРС, ожидаемые при упорядочении кислородных вакансий.

Строение решетки и сверхпроводящие свойства кристаллов $\mathrm{YBa}_{2} \mathrm{Cu}_{3} \mathrm{O}_{7-x}$ изменяются с изменением содержания кислорода [ $\left.{ }^{1}\right]$. Семи атомам кислорода в элементарной ячейке $(x=0)$ соответствует орторомбическая фаза (пространственная группа $D_{2 h}{ }^{1}$ ) с температурой сверхпроводящего перехода вблизи $93 \mathrm{~K}$. Тетрагональной фазе $(x=1$, пространственная группа $D_{4 h}{ }^{17}$ ) соответствует потеря атомов кислорода в позициях О(4), что уничтожает медно-кислородные цепочки $\ldots \mathrm{O}(4)-\mathrm{Cu}(1)-\mathrm{O}(4) \ldots$, а вместе с ними и сверхпроводящие свойства. Частичное упорядочение кислородных вакансий в $(a b)$ плоскостях предполагалось в связи с наблюдением еще одной сверхпроводящей фазы с температурой перехода вблизи $60 \mathrm{~K}\left[{ }^{2}\right]$. Методом электронной диффракции найдено сложное частичное упорядочение вакансий $\left[{ }^{3}\right]:$ удвоение элементарной ячейки вдоль оси $a$ при $x \approx 0,5$ и суперячейка размерами $2 \sqrt{2} a \times 2 \sqrt{2} b \times c$ при $x \approx 0,125$. В [ $\left.{ }^{4}\right]$ удалось получить высокую степень упорядочения вакансий с образованием новой фазы (орто-II), элементарная ячейка которой имеет размеры $2 a \times b \times c$ (рис. 1), причем температура сверхпроводящего перехода $53 \mathrm{~K}$.

В данной работе рассмотрены колебания обнаруженных сверхрешеток, вычислены симметризованные смещения ядер и обсуждены изменения в спектрах КРС, ожидаемые при упорядочении кислородных вакансий.

Рассмотрим элементарную ячейку фазы орто-II, изображенную на рис. 1. Она содержит 25 атомов, что соответствует стехиометрии $\mathrm{Y}_{2} \mathrm{Ba}_{4} \mathrm{Cu}_{6} \mathrm{O}_{13} \quad(x=0,5)$. Расположение атомов в слоях $\mathrm{Cu}(2)-\mathrm{O}(2)-$ -O(3) осталось таким же, как в простой решетке; из атомов $\mathrm{Cu}(1)$ каждый второй лишился кислорода О(4), так что цепочки $\mathrm{Cu}(1)-\mathrm{O}(4)$ чередуются с атомами $\mathrm{Cu}(1)$, соседствующими с вакансиями кислорода в позициях $\mathrm{O}(4)$ и О(5) и связанными только мостиковыми кислородами $\mathrm{O}(1)$. Симметрии позиций всех атомов расширенной элементарной ячейки приведены в таблице.

75 колебательных степеней свободы в Г-точке решетки орто-II преобразуются по неприводимым представлениям точечной группы $D_{2 h}$ следующим образом: $\quad 11 A_{g}+4 B_{1 g}+11 B_{2 g}+8 B_{3 g}+2 A_{u}+14 B_{1 u}+12 B_{2 u}+$ $+13 B_{3 u}$. 


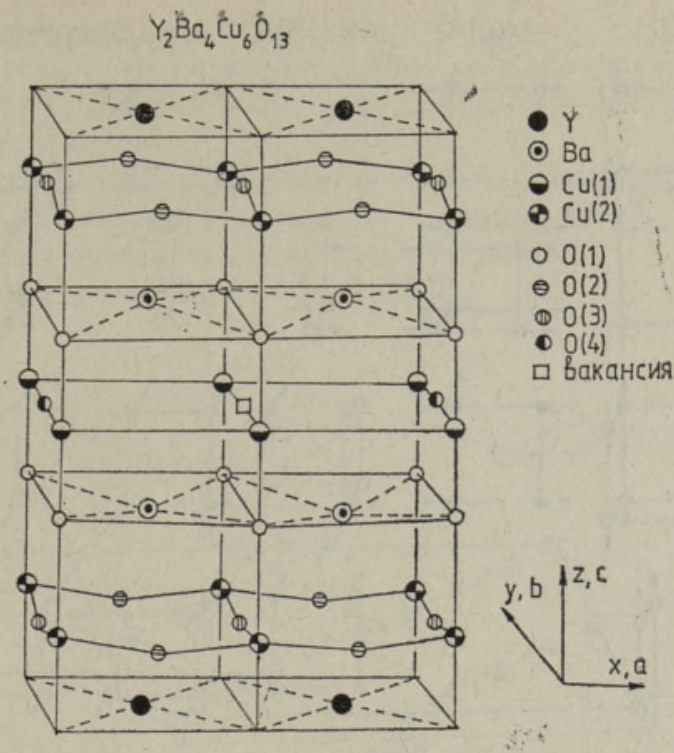

Рис. 1. Элементарная ячейка орто-II фазы кристалла $\mathrm{Y}_{1} \mathrm{Ba}_{2} \mathrm{Cu}_{3} \mathrm{O}_{6,5}$ с упорядоченными вакансиями.

Симметрия позиций атомов элементарной ячейки орто-II фазы кристалла $\mathrm{Y}_{1} \mathrm{Ba}_{2} \mathrm{Cu}_{3} \mathrm{O}_{6,5}$

\begin{tabular}{|c|c|c|c|c|}
\hline \multirow{2}{*}{ Атомы } & \multicolumn{3}{|c|}{ Число атомов } & \multirow{2}{*}{$\begin{array}{c}\text { Симметрия } \\
\text { позиции }\end{array}$} \\
\hline & на ребре & на грани & внутри & \\
\hline $\begin{array}{l}\mathrm{Y} \\
\mathrm{Ba} \\
\mathrm{Cu}(1) \\
\mathrm{Cu}(2) \\
\mathrm{O}(1) \\
\mathrm{O}(2) \\
\mathrm{O}(3) \\
\mathrm{O}(4)\end{array}$ & $\begin{array}{l}1 \\
2 \\
2\end{array}$ & $\begin{array}{l}2 \\
\\
1 \\
2 \\
2 \\
4 \\
2 \\
1\end{array}$ & 2 & $\begin{array}{l}\mathrm{C}_{2 v} \\
\mathrm{C}_{1} \\
\mathrm{D}_{2 h} \\
\mathrm{C}_{2 v} \\
\mathrm{C}_{2 v} \\
\mathrm{C}_{s} \\
\mathrm{C}_{2 v} \\
\mathrm{D}_{2 h}\end{array}$ \\
\hline
\end{tabular}

Рассмотрим полносимметричные смещения. В простой решетке имеется $5 \mathrm{~A}_{g}$ смещений, которые все направлены вдоль оси $c$ (z-смещения) $\left[{ }^{5}\right]$. Атомы расширенной ячейки совершают $8 z$-смещений и 3 $x$-смещения (рис. 2). Увеличение числа $z$-смещений обязано тому, что атомы, эквивалентные в простой ячейке, образуют две неэквивалентные группы в удвоенной ячейке. Например (см. рис. 1, 2), атомы $\mathrm{O}(1)$ в удвоенной ячейке образуют группу из $8 \mathrm{O}(1)$, расположенных на ребрах ячейки и вполне аналогичных атомам $\mathrm{O}(1)$ простой ячейки (частота соответствующей моды $\left.500 \mathrm{~cm}^{-1}\left[{ }^{6}\right]\right)$, а также группу $4 \mathrm{O}(1)$ на гранях, которые окружают вакансию. Под воздействием вакансии частота колебаний $\mathrm{O}(1)$ второй группы может измениться.

Появление четных полносимметричных $x$-смещений также обязано новым внутренним атомам. Удвоение ячейки вдоль направления $x$ привело к переходу точки $X$ на границе зоны Бриллюэна простой решетки в центр новой зоны. Например, $x$-смещение атомов $\mathrm{O}(2)$ (рис. 2) в Г-точке простой решетки могло быть только нечетным (это $B_{3 u}$-мода частотой $\left.555 \mathrm{~cm}^{-1}\left[{ }^{5}\right]\right)$; в удвоенной решетке возможны четные колебания этой группы атомов, частота которых должна быть близка к частоте соответствующей нечетной моды простой решетки. 


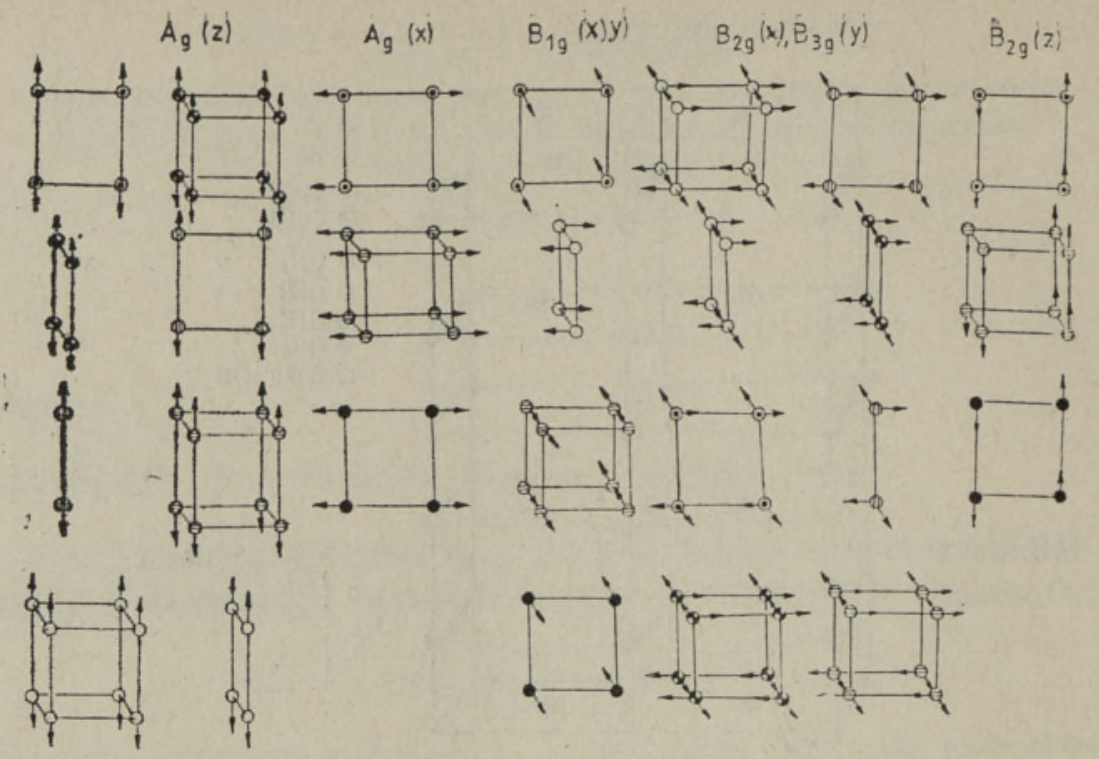

Рис. 2. Четные симметризованные смещения ядер элементарной ячейки орто-II фазы, дающие вклад в Г-точку зоны Бриллюэна.

Проведенный анализ показывает, что упорядочение вакансий кислорода с образованием фазы орто-ІІ возможно детектировать по изменению колебательного спектра $\mathrm{YBa}_{2} \mathrm{Cu}_{3} \mathrm{O}_{7-x}$. Рассмотрим отличия, ожидаемые в спектрах КРС при образовании фазы орто-II, опираясь на интерпретацию спектров КРС обычной орто-фазы, данную в $\left[{ }^{7,8}\right]$. Наиболее четкое различие состоит в появлении колебаний симметрии $B_{1 g}$, которые отсутствуют в случае разупорядочения вакансий. Четыре симметризованных смещения $B_{1 g}$ показаны на рис. 2. Тензор рассеяния для этих мод имеет недиагональные компоненты $\alpha_{x y}$, поэтому образование фазы орто-II можно детектировать по появлению спектра рассеяния от кристаллической плоскости $(a b)$ в перпендикулярной поляризации. Моды $B_{1 g}$ не имеют аналогов в простой решетке, однако, можно думать, что смещение тяжелого атома Ва дает низкочастотную моду в области 90-130 см$^{-1}$, где лежат остальные частоты колебаний Ва.

Полносимметричные $x$-смещения удвоенной ячейки должны обладать отличными от нуля компонентами тензора рассеяния $\alpha_{x x}$ и привести к появлению трех новых мод в спектре КРС, снятом от плоскости $(a b)$ в параллельной поляризации. По приведенным выше соображениям можно ожидать близости частот новых колебаний к частотам соответствующих нечетных колебаний симметрии $B_{3 u}$.

Еще одним отличительным признаком упорядочения вакансий может служить изменение контура полосы $500 \mathrm{~cm}^{-1}$, наиболее интенсивной полосы в спектре КРС в zz-поляризации. Во многих работах наблюдалось уширение этой полосы при появлении разупорядоченных вакансий кислорода [ $\left.{ }^{9}\right]$. Образование фазы орто-II должно сопровождаться сужением полосы $500 \mathrm{~cm}^{-1}$ и появлением второго компонента, по-видимому, со стороны низких частот.

Нами рассчитаны также симметризованные смещения для сверхрешетки с элементарной ячейкой $2 \sqrt{2} a \times 2 \sqrt{2} b \times c$, существование которой обсуждается в работе [3]. Такая сверхрешетка образуется, если вакансии кислорода занимают каждую четвертую позицию $\mathrm{O}(4)$ в 
каждой второй цепочке, как показано на рис. 3. Хотя упорядочения вакансий вдоль оси $c$ экспериментально не наблюдалось, мы идеализировали структуру, приняв расширенную элементарную ячейку, соответствующую стехиометрии $\mathrm{Y}_{8} \mathrm{Ba}_{16} \mathrm{Cu}_{24} \mathrm{O}_{55}$.

Эта элементарная ячейка также отвечает орторомбической симметрии и ее 309 колебательных степеней свободы распределяются по неприводимым представлениям следующим образом: $+35 B_{2 g}+37 B_{3 g}+27 A_{u}+44 B_{1 u}+45 B_{2 u}+43 B_{3 u}$.

$$
44 A_{g}+34 B_{1 g}+
$$
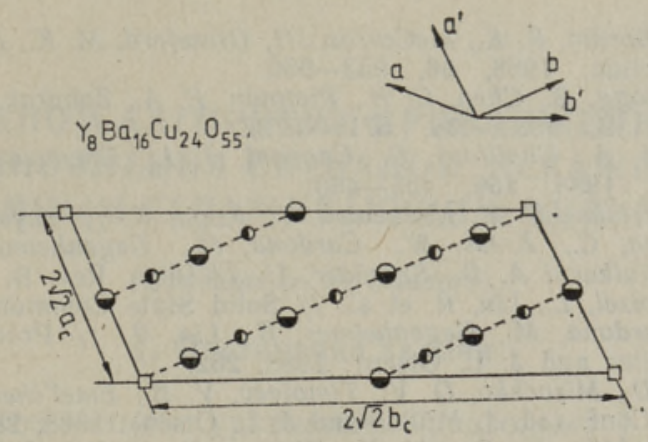

Рис. 3. $\mathrm{Cu}(1) \mathrm{O}(4)$ плоскость элементарной ячейки сверхрешетки кристалла $\mathrm{Y}_{1} \mathrm{Ba}_{2} \mathrm{Cu}_{3} \mathrm{O}_{6,875}$ с упорядоченными вакансиями.
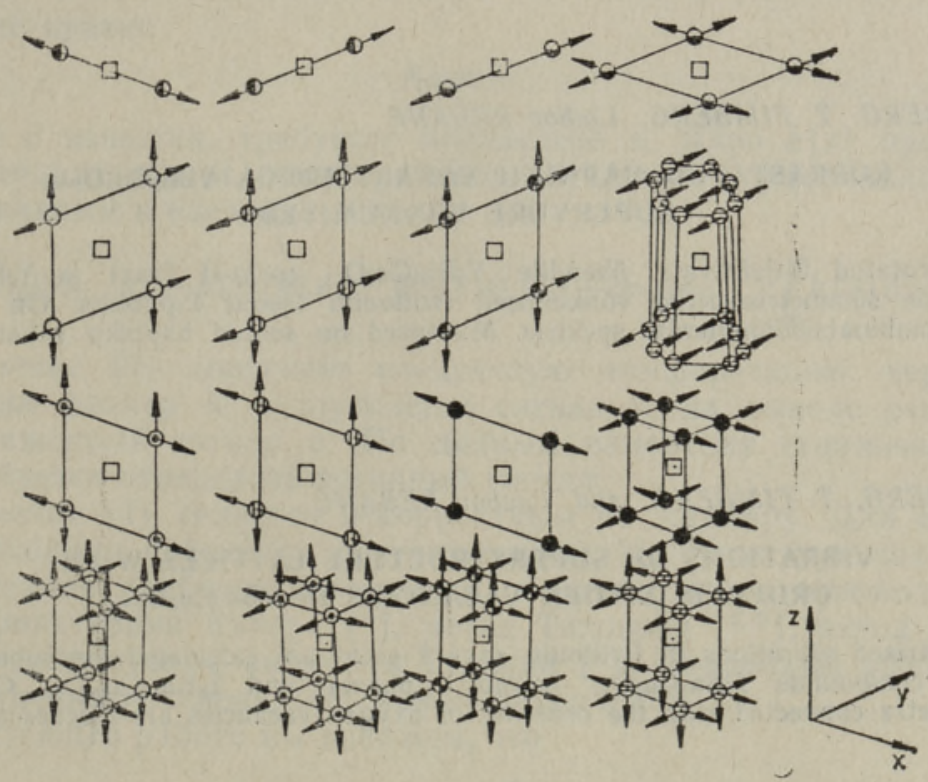

Рис. 4. Некоторые из дающих вклад в Г-точку зоны Бриллюэна полносимметричных смещений ядер сверхрешетки кристалла $\mathrm{Y}_{1} \mathrm{Ba}_{2} \mathrm{Cu}_{3} \mathrm{O}_{6,875}$ с упорядоченными вакансиями.

Поворот осей расширенной ячейки в плоскости $(a b)$ на $45^{\circ}$ приводит к появлению симметризованных смещений $x$ - и $y$-типа, показанных на рис. 4. При малой концентрации вакансий трудно ожидать существенного их влияния на частоты колебаний по сравнению с частотами колебаний простой решетки, однако появление колебаний $\mathrm{A}_{\mathrm{g}}(\mathrm{y})$ и связанных с ними компонентов тензора КРС $\alpha_{y y}$ возможно выделить, особенно в спектрах монокристаллических образцов лишенных двойникования. 
Приведенные выше рассуждения могут быть экंсперимента̇лььно про́верены лишь на образцах, обладающих высокой степенью упорядочения вакансий, для достижения которой требуется отработка режимов дополнительной температурной тренировки образцов $\left[{ }^{4}\right]$.

\section{ЛИТЕРА Т У Р А}

1. Farneth, W. E., Bordia, R. K., McCarron III, Grawford, M. K., Flippen, R. B. // Solid State Commun, 1988, 66, 953-960.

2. Cava, R. J., Batlogg, B., Chen, C. H., Rietman, E. A., Zahurak, S. M., Werder, D. // Phys. Rev. B, 1987, 36, 5719-5722.

3. Alario-Franco, M. A., Chaillout, C., Capponi, J. J., Chenavas, J., Marezio, M. // Physica C. $1988,156,455-460$.

4. Takabatake, T., Ishikawa, M., Nakazawa, Y., Koga, K. // Physica C, 1988, 152, 424.

5. Liu, R., Thomsen, C., Kress, W., Cardona, M., Gegenheimer, B., Wette, F. W., Prade, J., Kulkarni A. D., Schröder, U. // Phys. Rev. B, 1988, 37, 7971-7974.

6. Cardona, M., Genzel, L., Liu, R. et al. // Solid State Commun, 1987, 64, 727.

7. Thomsen, C., Cardona, M., Gegenheimer, B., Liu, R. // Proc. of Interlaken Conf. (ed. J. Müller and J. L. Olsen). 1988, 262.

8. Kulakovskii, V. D., Misochko, O. V., Timofeev, V. B., Emel'chenke, G. A. // Proc. of Interlaken Conf. (ed. J. Müller and J. L. Olsen). 1988, 286.

9. Nishitani, R., Yoshida, N., Sasaki, Y., Nishina, Y. // Jap. J. Appl. Phys., 1988, 27, L1284-L1286.

Ннститут химической и биологической физики Академии наук Эстонской ССР

Поступила в редакцию $16 /$ XII 1988

G. BLUMBERG, T. FIMBERG, Ljubov REBANE

\section{KORRASTATUD HAPNIKU VAKANTSIDEGA $\mathrm{YBa}_{2} \mathrm{Cu}_{3} \mathrm{O}_{7-x}$ SUPERVORE VONKUMISED}

On arvutatud ülijuhtivate ühendite $\mathrm{Y}_{2} \mathrm{Ba}_{4} \mathrm{Cu}_{6} \mathrm{O}_{13}$ (orto-II faas) ja $\mathrm{Y}_{6} \mathrm{Ba}_{16} \mathrm{Cu}_{24} \mathrm{O}_{55}$ kristallvōrede sümmetriseeritud võnkumised Brillouini tsooni $\Gamma$-punktis. On vaadeldud muutusi kombinatsioonhajumise spektris. Muutused on seotud hapniku vakantside korrastusega.

G. BLUMBERG, T. FIMBERG, and Lyubov REBANE

\section{VIBRATIONS ŌF SUPERSTRUCTURE LATTICES WITH ORDERED OXYGEN VACANCIES IN $\mathrm{YBa}_{2} \mathrm{Cu}_{3} \mathrm{O}_{7-x}$}

Symmetrized vibrations in Brillouin zone $\Gamma$-point are calculated for superconductor crystalline compounds $\mathrm{Y}_{2} \mathrm{Ba}_{4} \mathrm{Cu}_{6} \mathrm{O}_{13}$ (ortho-II phase) and $\mathrm{Y}_{8} \mathrm{Ba}_{16} \mathrm{Cu}_{24} \mathrm{O}_{55}$. Changes in Raman spectra connected with the ordering of oxygen vacancies are discussed. 